Research Paper

\title{
Combining Red Blood Cell Distribution Width (RDW-CV) and CEA Predict Poor Prognosis for Survival Outcomes in Colorectal Cancer
}

\author{
Yalun $\mathrm{Li}^{1}$, Chengzhong Xing1, Minjie Wei2 ${ }^{2}$ Huizhe $\mathrm{Wu}^{2}$, Xiaoyun $\mathrm{Hu}^{2}$, Shanqiong $\mathrm{Li}^{2}$, Guangwei Sun${ }^{1}$, \\ Guangzhe Zhang ${ }^{1}$, Bo Wu ${ }^{1}$, Fangxiao Zhang ${ }^{3}$, Zhuang $\mathrm{Li}^{1 凶}$ \\ 1. Department of Anorectal Surgery, First Affiliated Hospital of China Medical University, Shenyang, Liaoning, China \\ 2. Department of Pharmacology, School of Pharmacy, China Medical University, Shenyang, Liaoning, China \\ 3. Department of Intensive Care Unit, First Affiliated Hospital of China Medical University, Shenyang, Liaoning, China \\ $\square$ Corresponding author: Zhuang Li, Department of Anorectal Surgery, First Affiliated Hospital of China Medical University, Shenyang, Liaoning, China. Tel: \\ +86-13840389895; E-mail: cmu1stbill@163.com \\ (c) Ivyspring International Publisher. This is an open access article distributed under the terms of the Creative Commons Attribution (CC BY-NC) license \\ (https://creativecommons.org/licenses/by-nc/4.0/). See http://ivyspring.com/terms for full terms and conditions.
}

Received: 2018.08.05; Accepted: 2019.01.04; Published: 2019.01.29

\begin{abstract}
Background: Colorectal cancer is one of the common tumors that seriously threaten human health worldwide. Serum tumor markers, including CEA and CA19-9, have become the focus of research on colorectal cancer in recent years. As one of the classic blood test results, RDW is related to the pathological features, diagnosis and prognosis of various cancers in recent studies. We hope to search the correlation between RDW and the pathological features of colorectal cancer through the following studies, explore the potential relationship between RDW and the prognosis of colorectal cancer, and find a more effective prognostic evaluation method by combining other blood markers.

Methods: We retrospectively analyzed 168 patients with colorectal cancer included in this study, collected their clinical data, tumor pathological features and their preoperative blood test results including RDW value and tumor markers, and grouped them. After 3 and 5 years of follow-up, the recurrence and survival status were defined, and the above data were statistically analyzed.

Results: The distribution frequency/rate of abnormal RDW-CV in colorectal cancer patients was significantly increased in the elderly $(>62)$, colon cancer, serosal permeability, lymph node metastasis, stage III and IV, peripheral adhesion ( $<<0.05)$. Furthermore, RDW-CV was significantly positively correlated with abnormal high values of tumor serum markers CEA and CA19-9 $(P<0.05)$. More importantly, ROC curve analysis found that the abnormal increase in RDW-CV in colorectal cancer was associated with the shortening of DFS and OS in patients who were followed up for 3 and 5 years $(P<$ 0.05). Further combined with CEA, it was found that the prognosis and survival of patients with colorectal cancer in 3 and 5 years were more accurate and effective than independent prediction (AUC of DFS in $3 / 5$ years $=0.630 / 0.635$, AUC of OS in $3 / 5$ years $=0.692 / 0.652$ ).

Conclusion: RDW-CV is correlated with the pathological features of colorectal cancer, indicating a worse malignant tendency of tumor. RDW-CV can independently evaluate the prognosis of colorectal cancer patients, and combined with the high value of CEA, it can effectively indicate the adverse recurrence and survival prognosis.
\end{abstract}

Key words: RDW, CEA, prognosis, colorectal cancer

\section{Introduction}

Blood routine is one of the commonly used test methods in clinical practice, in which the red blood cell distribution width (RDW-CV) is an important classic item of blood routine examination, which mainly reflects the evenness of the volume and size of red blood cells. [1] The measurement of RDW is often indicated by two indicators, RDW-CV (RBC distribution width-variation coefficient) and RDW-SD 
(RBC distribution width-standard deviation), which can be applied to the testing of different diseases as required. In the past, RDW was mainly used to diagnose different types of anemia, hematopoietic disorder, congenital erythrocyte abnormalities and other blood system diseases. Meanwhile, it has been gradually studied in other disease-related fields in recent years. [2-5]

Colorectal cancer is one of the most common malignant tumors in the world, which seriously harms human health. Even with the continuous exploration of its research, the disease has not been fully understood. [6, 7] The evaluation of malignancy degree and prognosis of colorectal cancer is of great significance in clinical work. At present, the evaluation of malignancy degree of colorectal cancer mainly comes from TNM stage, which refers to some pathologic indicators after surgery, including whether to penetrate serosa, lymph node metastasis, other organ metastasis and peripheral adhesion. [8, 9] Although these evaluation indicators have the advantages of definite stages and specific description, most of them come from postoperative pathological reports and require pathological examination after tumor resection, which has the disadvantage of judging lag. According to previous studies, malignant tumors are diseases that can lead to changes in the blood status of the whole body, rather than only local lesions. ${ }^{[10-12]}$ Classical tumor stage is more targeted at the evaluation of the tumor lesion itself, and the inability to evaluate the patient's systemic status is also one of its defects.

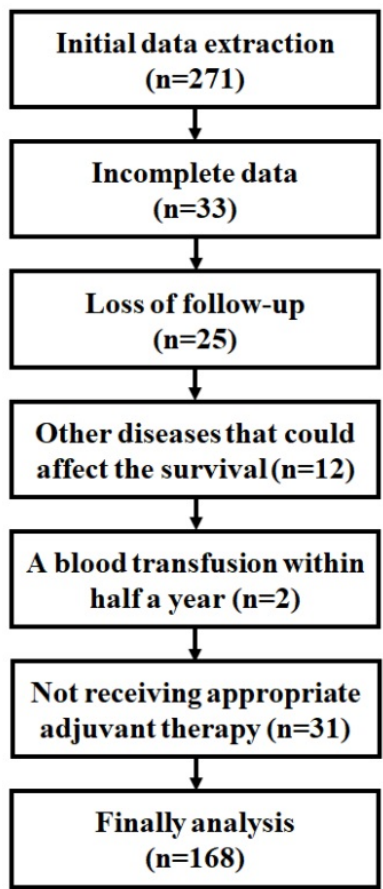

Figure 1. Screening process of sample patients
Tumor markers in the blood (such as CEA, CA19-9, CA12-5, etc.) are also considered to play an important role in diagnosis, malignant evaluation and prognosis prediction. [13] Because of the advantages of easy access to blood samples, low trauma, long-term monitoring, and assessment of systemic status, they are increasingly valued. By unceasingly exploring new blood indicators, more accurate and comprehensive assessment of the degree of malignancy and prognosis of colorectal cancer is of great guiding significance to clinical work. It has been reported that the RDW level of many patients with malignant tumors, including colorectal cancer, has been widely discussed, proving that the level of RDW in patients' blood is correlated with the diagnosis, staging and prognosis. [14-17] Therefore, we hope to analyze the relationship between RDW and tumor malignancy in colorectal cancer through the following retrospective study and explore its potential to be a more effective new prognostic evaluation blood indicator.

\section{Materials and Methods}

\section{Ethics statement}

The First Hospital of China Medical University and the Medical Ethics Committee of China Medical University approved this study. Due to the retrospective nature of the study, the First Hospital of China Medical University and the Medical Ethics Committee of China Medical University waived the need of written informed consent by the patients. All the samples were anonymous.

\section{Patients and Methods}

Study Population: From October 2012 to July 2013, 271 patients received surgical treatment for colorectal cancer in the anorectal surgery department of the First Affiliated Hospital of China Medical University were collected, and 168 patients required for the study were obtained after the following standard screening as discovery phase samples (Figure 1). The exclusion criteria: 1) Incomplete patients information and/or loss of follow-up, 2) Patients with severe cardiovascular and cerebrovascular diseases, pulmonary diseases, blood diseases, infectious diseases and other malignant tumors that may affect survival rate, such as cerebral infarction, pulmonary infarction, high risk of hypertension or HIV infection, etc, 3)Blood transfusion was performed half a year before the operation, 4)Patients who did not received appropriate adjuvant therapy based on NCCN guideline ${ }^{[18,19]}$. Final patients sample include 54 colon cancers and 114 rectal cancers. 
Blood Biochemistry: All blood samples were collected one week before the operation and examined by laboratory department of the First Affiliated Hospital of China Medical University through blood cell analyzer (Sysmex XE-5000, Japan) and Automatic chemiluminescence immunoanalyzer (Roche, Cobas E601, Switzerland). The report results of blood routine test and tumor marker test included: RDW-CV, RDW-SD, CEA, CA12-5, CA19-9 and other relevant indicators. According to the normal range of each indicator, the patients were divided into two groups: "Normal group" and "Abnormal group". The abnormal levels of RDW, CEA, CA19-9 and CA12-5 were all higher than the normal value.

Tissue Pathology: Postoperative complete resection of tumor specimens was sent to the second tumor institute of the First Affiliated Hospital of China Medical University for Pathological Examination. The tumor growth pattern, lymph node metastasis, infiltration depth, pathological type and differentiation degree were reported by qualified professional pathologists. According to the latest AJCC cancer manual[20], TNM was re-staged and grouped.

Methods: Complete clinical and pathological characteristics of the sample patients were collected for collation and statistical analysis, including gender, age, family history, smoking history, alcoholism history, blood biochemical results and pathological description. All patients were required to follow-up and review strictly after treatment. Followed-up with 3 years and 5 years after surgery for tumor recurrence and survival were acquired.

\section{Statistical analysis}

Statistical analysis was conducted by SPSS 24.0 (Chicago, IL, USA) and Graphpad Prism 7.0 (Graphpad Software, CA, USA). The classification data were analyzed using the Pearson chi-square test, continuous variables were tested by spearman two-variable correlation test and the linearity was analyzed by the log-rank test. ROC curve was used to evaluate the sensitivity, specificity and the area of AUC. A $P$ value of less than $0.05(P<0.05)$ was considered statistically significant.

\section{Results}

\section{Baseline characteristics}

According to statistics, a total of 168 patients were studied, with an average age of 61.92 years. Including 94 males and 74 females, 54 cases of colon cancer and 114 cases of rectal cancer, only 23 patients had a family history of malignant tumors. 41 patients had the habit of smoking for a long time. 19 patients had light drunk daily, and 8 patients had alcohol abuse all year round. According to pathological characteristics: There were 81 cases of nests growth and 87 cases of infiltrating growth in the tumor growth pattern, meanwhile there were 26, 96 and 46 cases of poor, moderate and high differentiation.141 cases of adenocarcinoma accounted for most samples. Most of the samples were pathologically penetrating serosa (109 cases), without lymph node metastasis (100 cases), and no other viscera metastases (164 cases) and adjacent viscera adhesion (141 cases) were detected in surgery. TNM I, II, III, IV stage respectively had 28, 67, 69, 4 cases (Table S1). The results of the blood test showed: The average RDW-CV was 87.7 [61.8-99.2] \%, of which 122 cases were in the normal range and the remaining 46 cases were higher than the normal high value. The RDW-SD average was 43.7 [36.0-77.0] fL, of which 116 were in the normal range and the remaining 52 cases were above the normal range. The mean levels of tumor markers in test of CEA, AFP, CA12-5 and CA19-9 were $6.90[0.12-59.53] \mathrm{ng} / \mathrm{ml}, 3.40[0.96-11.13] \mathrm{ng} / \mathrm{ml}$, $13.41[0.81-81.68] \mathrm{U} / \mathrm{ml}$ and $28.33[0.60-736.70] \mathrm{U} / \mathrm{ml}$. According to the range of normal values, 85, 9, 7, 34 cases were higher than normal maximum respectively. After follow-up, tumor progression was found in 33 patients and 17 patients died 3 years after surgery. Five years after surgery, 51 patients developed tumors progression and 41 died (Table 1).

Table 1. Blood biochemical examination results of colorectal cancer patients

\begin{tabular}{lll}
\hline Groups & Normal range & Data \\
\hline RDW-CV [average, range](\%) & {$[10.8-14.5]$} & {$[87.7,61.8-99.2]$} \\
Normal & & 122 \\
Abnormal & & 46 \\
RDW-SD [average, range](fL) & {$[36.0-46.0]$} & {$[43.7,36.0-77.0]$} \\
Normal & & 116 \\
Abnormal & & 52 \\
CEA [average, range](ng/ml) & {$[0.00-4.30]$} & {$[6.90,0.12-59.53]$} \\
Normal & & 83 \\
Abnormal & & 85 \\
AFP [average, range](ng/ml) & {$[0.00-7.00]$} & {$[3.40,0.96-11.13]$} \\
Normal & & 159 \\
Abnormal & 9 \\
CA12-5 [average, range](U/ml) & {$[0.00-35.00]$} & {$[13.41,0.81-81.68]$} \\
Normal & & 161 \\
Abnormal & 7 \\
CA19-9 [average, range](U/ml) & {$[0.00-27.00]$} & {$[28.33,0.60-736.70]$} \\
Normal & & 134 \\
Abnormal & & 34 \\
3 years DFS & & \\
No-Progress & & 135 \\
Progress & 33 \\
5 years DFS & \\
No-Progress & \\
Progress & & 117 \\
3 years OS & 51 \\
Survive & \\
Dead & & 151 \\
5 years OS & & 17 \\
Survive & & \\
Dead & & 127 \\
\hline
\end{tabular}




\section{Correlation between RDW-CV/SD and characteristics}

According to different characteristics, we divided the sample patients into groups and analyzed the correlation between RDW-CV and them in turn (Figure 2). Chi-square test and logistic regression analysis using age, gender and family history as covariate reference factors to analyze the correlation between RDW-CV/SD and various clinical and pathological indicators: In the blood biochemical tests, RDW-CV was positively correlated with the results of CEA and CA19-9 classification variables $(P<0.05)$, and only the continuous variables of CEA were linearly correlated $(P<0.01)$ (Figure 3 ).

a
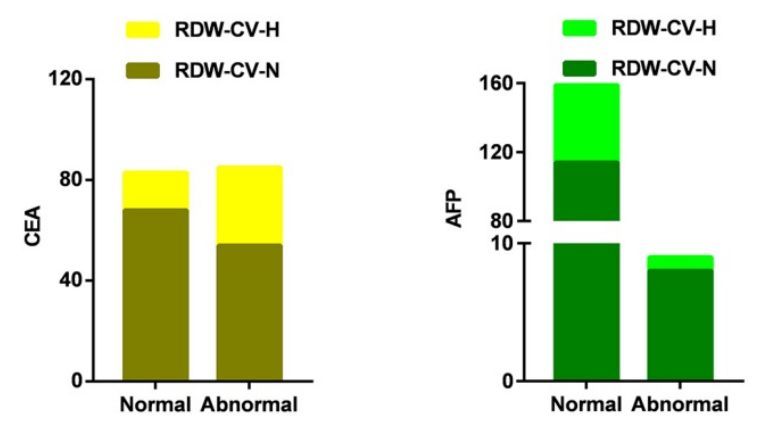

b
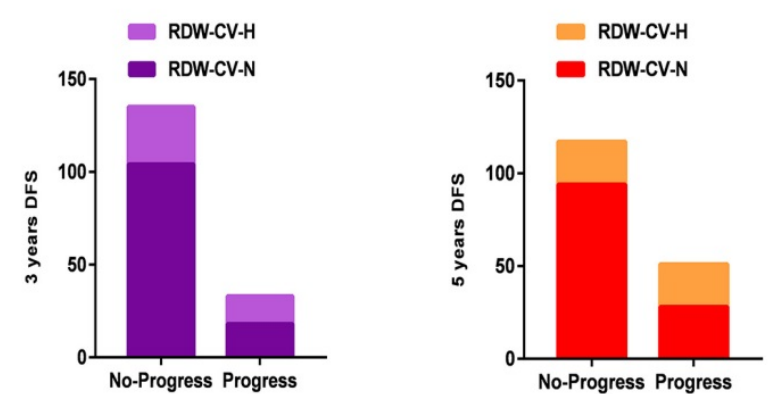

In the results of analyzing the survival status, RDW-CV has a clear linear correlation with DFS and OS at both time points of 3 and 5 years and the sample population with high RDW-CV is more likely to have poor prognosis of disease progression or death at both time points of follow-up $(\mathrm{P}<0.05)$. RDW-SD also conducted correlation analysis of the above indicators, and the results showed that there was no clear correlation between RDW-SD and DFS in terms of prognosis, but in OS prognosis analysis of 3 and 5 years, abnormal high RDW-SD indicated poor OS prognosis $(\mathrm{P}<0.05)$ (Table 2).
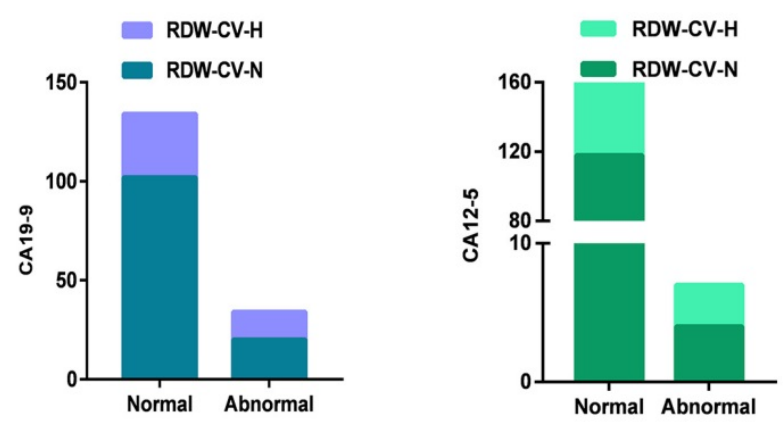

Figure 2. Distribution of RDW-CV in different biochemical indexes and prognosis. (a) Blood biochemical indexes results (b) Prognosis results

a

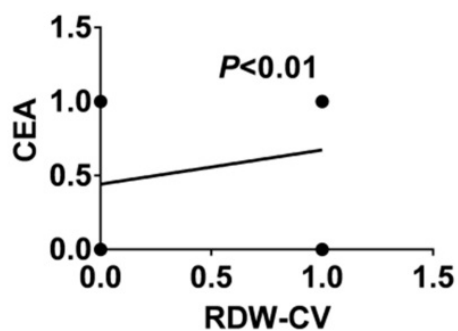

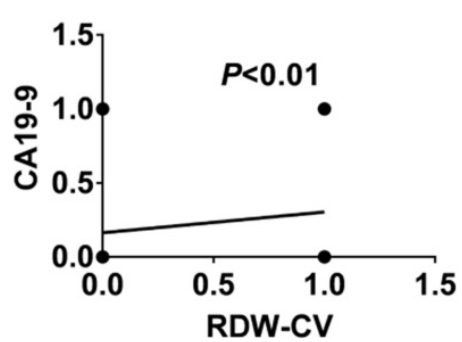

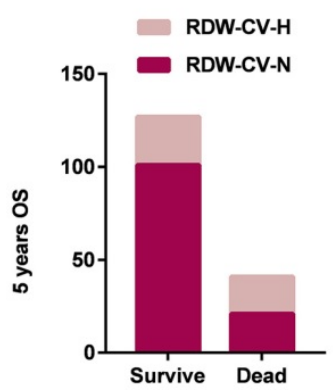

Figure 3. RDW-CV and blood biochemical indexes analyzed by linear regression analysis. (a) Classified data for CEA and CA19-9 with RDW-CV (b) Continuous data for CEA with RDW-CV 
Table 2. Correlation of RDW-CV and RDW-SD with blood biochemical indexes and prognosis of colorectal cancer patients.

\begin{tabular}{|c|c|c|c|c|c|c|c|c|}
\hline & RDW-CV & & & & RDW-SI & & & \\
\hline Characteristics & Normal & High & $P † \ddagger$ & Adjusted OR(95\%CI) § & Normal & High & $P \dagger \ddagger$ & Adjusted OR(95\%CI) $\S$ \\
\hline \multicolumn{9}{|l|}{ CEA } \\
\hline Normal & 68 & 15 & $0.008 \dagger$ & 1.000 & 62 & 21 & $0.117 \dagger$ & 1.000 \\
\hline Abnormal & 54 & 31 & $0.016 \ddagger$ & 2.475 (1.187-5.159) & 54 & 31 & $0.194 \ddagger$ & $1.568(0.795-3.091)$ \\
\hline \multicolumn{9}{|l|}{ AFP } \\
\hline Normal & 114 & 45 & $0.261 \dagger$ & 1.000 & 109 & 50 & $0.560 \dagger$ & 1.000 \\
\hline Abnormal & 8 & 1 & $0.496 \ddagger$ & $0.474(0.055-4.079)$ & 7 & 2 & $0.646 \ddagger$ & $0.679(0.131-3.534)$ \\
\hline \multicolumn{9}{|l|}{ CA12-5 } \\
\hline Normal & 118 & 43 & $0.348 \dagger$ & 1.000 & 110 & 51 & $0.330 \dagger$ & 1.000 \\
\hline Abnormal & 4 & 3 & $0.301 \ddagger$ & $2.347(0.466-11.820)$ & 6 & 1 & $0.260 \ddagger$ & $0.288(0.033-2.520)$ \\
\hline \multicolumn{9}{|l|}{ CA19-9 } \\
\hline Normal & 102 & 32 & $0.043 \dagger$ & 1.000 & 96 & 38 & $0.149 \dagger$ & 1.000 \\
\hline Abnormal & 20 & 14 & $0.048 \ddagger$ & $2.219(1.006-4.894)$ & 20 & 14 & $0.208 \ddagger$ & $1.665(0.753-3.684)$ \\
\hline \multicolumn{9}{|l|}{3 years DFS } \\
\hline No-Progress & 104 & 31 & $0.009+$ & 1.000 & 96 & 39 & $0.242 \dagger$ & 1.000 \\
\hline Progress & 18 & 15 & $0.015 \ddagger$ & $2.800(1.219-6.433)$ & 20 & 13 & $0.358 \ddagger$ & $1.460(0.652-3.270)$ \\
\hline \multicolumn{9}{|l|}{5 years DFS } \\
\hline No-Progress & 94 & 23 & $0.001 \dagger$ & 1.000 & 85 & 32 & $0.126 \dagger$ & 1.000 \\
\hline Progress & 28 & 23 & $0.001 \ddagger$ & $3.586(1.660-7.748)$ & 31 & 20 & $0.224 \ddagger$ & $1.565(0.761-3.221)$ \\
\hline \multicolumn{9}{|l|}{3 years OS } \\
\hline Survive & 115 & 36 & $0.002 \dagger$ & 1.000 & 108 & 43 & $0.039 \dagger$ & 1.000 \\
\hline Dead & 7 & 10 & $0.002 \ddagger$ & $5.898(1.951-17.828)$ & 8 & 9 & $0.036 \ddagger$ & $3.066(1.074-8.757)$ \\
\hline \multicolumn{9}{|l|}{5 years OS } \\
\hline Survive & 101 & 26 & $0.000 \dagger$ & 1.000 & 94 & 33 & $0.014 \dagger$ & 1.000 \\
\hline Dead & 21 & 20 & $0.000 \ddagger$ & 4.433 (1.923-10.219) & 22 & 19 & $0.029 \ddagger$ & $2.353(1.092-5.069)$ \\
\hline
\end{tabular}

tP values were calculated from 2 -sided chi-square tests.

$\ddagger \mathrm{P}$ values were calculated by unconditional logistic regression adjusted for age, gender and family history.

sOR and $95 \% \mathrm{CI}$ values were calculated by unconditional logistic regression adjusted for age, gender and family history.

In addition, we found that high RDW-CV were more likely to be obtained in older age, colon cancer and non-adenocarcinoma patients. The distribution of patients with high RDW-CV increased in patients with the following characteristics: serosa infiltration, lymph node metastasis and adhesion with surrounding tissues or organs. No linear correlation was found with TNM stage, but according to the period of stage I + II, III + IV groups, we found that higher RDW-CV was more likely to be detected in patients with advanced stages $(P<0.05)$. Patients with high RDW-SD were only more likely to develop non-adenocarcinoma colon tumors, including mucinous adenocarcinoma or signet ring cell carcinoma (Table S2).

\section{Comparison between RDW-CV, RDW-CV combined with CEA/CA19-9 and other indicators in prediction of cancer prognosis}

According to the above results, we believe that $\mathrm{RDW}-\mathrm{CV}$ is a potential indicator to describe the malignancy of colorectal cancer, so we conducted the following statistical analysis by the ROC curve of follow-up prognosis in the discovery phase samples: TNM stages showed the highest correlation in 3 and 5 years DFS/OS poor prognosis (AUC $=0.768,0.719$, $0.748,0.673, P<0.05)$ and older people were more likely to have a dismal 5 years DFS and OS prognosis (AUC $=0.603,0.605, P<0.05)$. RDW-CV might not only be used as an independent predictor to evaluate the prognosis of DFS and OS in 3 and 5 years
(AUC $=0.612,0.627,0.675,0.642, P<0.05$ ), but also patients with abnormal high value of both RDW-CV and CEA can obtain poor prognosis results $(P<0.05)$. The AUC area of DFS-related ROC curves in 3 and 5 years were 0.630 and 0.635 , and that of OS-related ROC curves were 0.692 and 0.652 , indicating higher sensitivity and specificity of adverse prognosis. However, the abnormal expression of RDW-CV combined with CA19-9, gender, differentiation and pathological pattern had no ideal statistical result (Figure 4, Table 3, Table S3).

Based on the main finding above, then we collected and followed 49 patients' information in August, September and October 2013 as replication phase samples through the same exclusion criteria to verify the main findings of previous study. The similar results were found in ROC curve: In the replication phase samples, $\mathrm{RDW}-\mathrm{CV}$, RDW-CV combined with CEA and TNM all showed definite correlation with poor DFS/OS prognosis. The AUC area of TNM staging was the largest $(\mathrm{AUC}=0.892$, $0.794,0.832,0.784, P<0.05)$, and compared with $\mathrm{RDW}-\mathrm{CV}$ as an independent factor (AUC $=0.723$, $0.718,0.774,0.724, P<0.05)$, RDW-CV combined with CEA had a larger AUC area in ROC curve associated with poor prognosis (AUC $=0.762,0.720,0.810,0.765$, $P<0.05)$, which confirmed the finding of previous studies: RDW-CV combined with CEA was more effective in warning of tumor recurrence and death after surgery. Such indicators as RDW-CV combined with CA19-9, differentiation, and pathological type 
still had no statistical significance in the evaluation of prognosis. The higher age only showed a correlation with $O S$ at the time point of 5 years in replication phase samples $(\mathrm{AUC}=0.742, P<0.05)$ (Figure 4, Table 3, Table S3).

\section{Discussion}

RDW, as one of the classical evaluation indexes for the degree of erythrocyte morphology imbalance in blood, can effectively reflect the heterogeneity of erythrocyte volume, and is usually used for the evaluation of blood diseases, cardiovascular diseases and infectious diseases. [21-24] In recent years, it has been found that its value is related to the early diagnosis of tumor, but whether it can be used as a new molecular marker for early warning and prognosis evaluation of cancer remains to be studied. $\mathrm{RDW}-\mathrm{CV}$ can independently evaluate the prognosis of colorectal cancer patients, and combined with the high value of CEA, it can effectively indicate the adverse recurrence and survival prognosis.
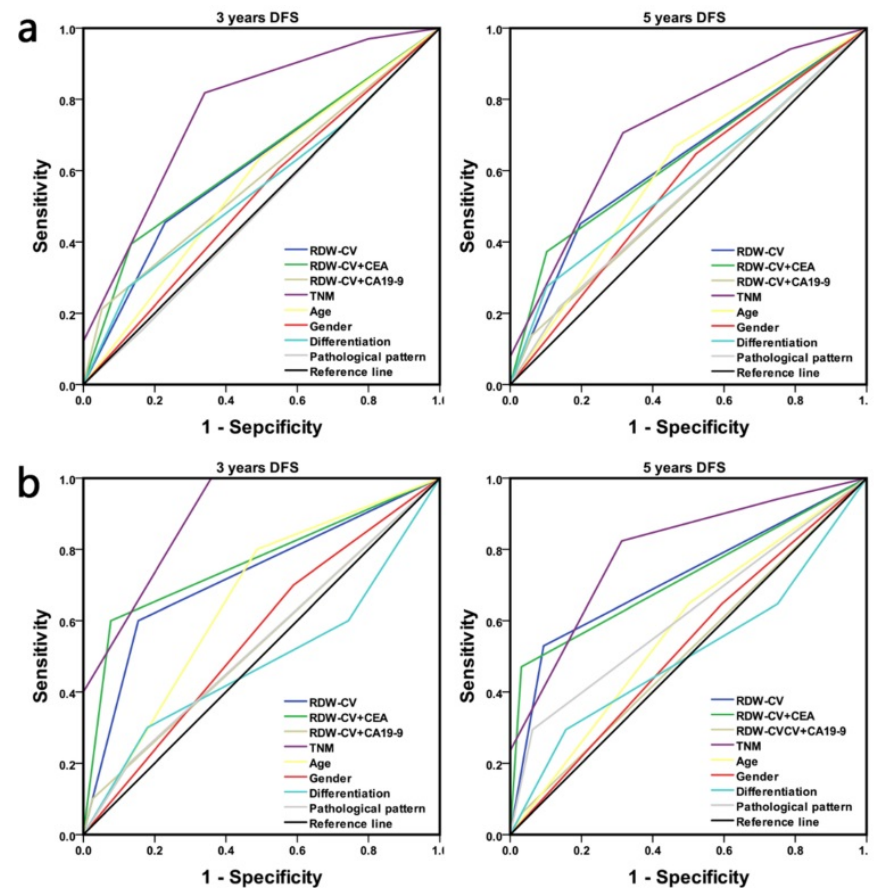

RDW values can be reflected by RDW-CV and RDW-SD values that could indicate the existence of mixed cell groups with unequal RBC size. The results of our study showed that RDW-CV was significantly correlated with the pathological features and prognosis of cancers, while the suggestive effect of RDW-SD was not obvious, so we focused on $\mathrm{RDW}-\mathrm{CV}$. We found that high RDW-CV was more common in colorectal cancer patients with non-adenocarcinoma pathology types such as mucinous adenocarcinoma or signet ring cell carcinoma. Pathologic prognostic survival analysis generally considers that non-adenocarcinoma colorectal cancer has higher grade of malignancy and lower survival rate than adenocarcinoma, therefore, we conclude that RDW-CV may be associated with poor prognosis. ${ }^{[25-28]}$ Then we studied the relationship between RDW-CV and cancer pathological indicators and found that patients with high RDW-CV were more likely to have malignant features of infiltration of serosa, lymph node metastasis and adhesion to surrounding tissues and organs.
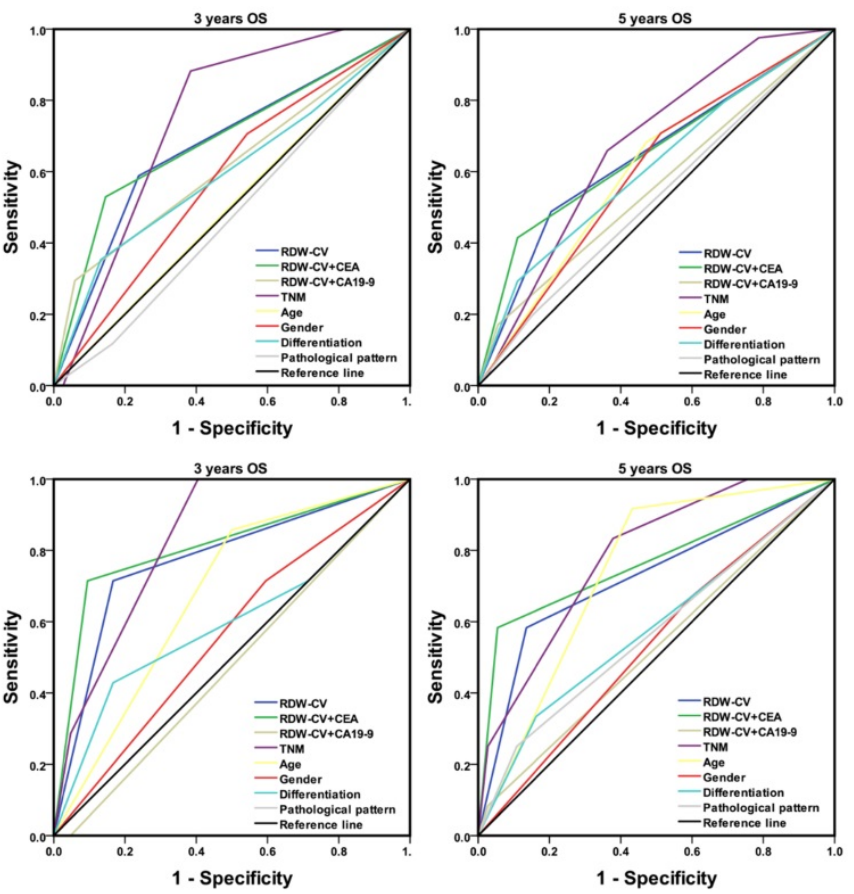

Figure 4. Prognosis survival ROC curve of RDW-CV, RDW-CV with CEA/CA19-9 and other indicators. The sensitivity and specificity for each outcome were calculated. (a) 3 and 5 years follow-up of DFS/OS in discovery phase samples (b) 3 and 5 years follow-up of DFS/OS in replication phase samples.

Table 3. The AUC area and $P$ value of DFS/OS at 3 years and 5 years by different indicators

\begin{tabular}{|c|c|c|c|c|c|c|c|c|c|c|c|c|c|c|c|c|}
\hline \multirow[t]{3}{*}{ Groups } & \multicolumn{8}{|c|}{ Discovery phase samples } & \multicolumn{8}{|c|}{ Replication phase samples } \\
\hline & \multicolumn{2}{|c|}{3 years DFS } & \multicolumn{2}{|c|}{5 years DFS } & \multicolumn{2}{|c|}{3 years OS } & \multicolumn{2}{|c|}{5 years OS } & \multicolumn{2}{|c|}{3 years DFS } & \multicolumn{2}{|c|}{5 years DFS } & \multicolumn{2}{|c|}{3 years OS } & \multicolumn{2}{|c|}{5 years OS } \\
\hline & AUC & $P \dagger$ & AUC & $P \dagger$ & AUC & $P \dagger$ & AUC & $P \dagger$ & AUC & $P \dagger$ & AUC & $P \dagger$ & AUC & $P \dagger$ & AUC & $P \dagger$ \\
\hline RDW-CV & 0.612 & 0.045 & 0.627 & 0.009 & 0.675 & 0.018 & 0.642 & 0.006 & 0.723 & 0.031 & 0.718 & 0.013 & 0.774 & 0.021 & 0.724 & 0.021 \\
\hline $\mathrm{RDW}-\mathrm{CV}+\mathrm{CEA}$ & 0.630 & 0.020 & 0.635 & 0.005 & 0.692 & 0.010 & 0.652 & 0.003 & 0.762 & 0.011 & 0.720 & 0.012 & 0.810 & 0.009 & 0.765 & 0.006 \\
\hline RDW-CV+CA19-9 & 0.580 & 0.154 & 0.539 & 0.426 & 0.617 & 0.113 & 0.558 & 0.266 & 0.537 & 0.719 & 0.514 & 0.875 & 0.476 & 0.841 & 0.528 & 0.771 \\
\hline TNM & 0.768 & 0.000 & 0.719 & 0.000 & 0.748 & 0.001 & 0.673 & 0.001 & 0.892 & 0.000 & 0.794 & 0.001 & 0.832 & 0.005 & 0.784 & 0.003 \\
\hline Age & 0.570 & 0.213 & 0.603 & 0.035 & 0.503 & 0.966 & 0.605 & 0.043 & 0.656 & 0.130 & 0.574 & 0.401 & 0.679 & 0.134 & 0.742 & 0.012 \\
\hline
\end{tabular}

tP values were calculated from ROC curve. 
Above these malignant characteristics were important parts of TNM staging, comprehensive analysis and judgment found that stage III and IV RDW - CV of colorectal cancer was higher than I and II stage, and this result was supported by study from Yang D, et al. [29] Adhesion is also an important indication of malignancy in colorectal cancer. Similar findings are showed in other cancers. Kemal $\mathrm{Y}$ et al tested RDW levels in the blood of patients with endometrial cancer and found that their values were correlated with pathological characteristics. [30] Therefore, we concluded that RDW-CV might be an effective indicator to reflect the malignant degree of colorectal cancer.

As classic evaluation factors, tumor markers such as CEA and CA19-9 were not only related to the development degree of colorectal cancer, but also directly related to tumor recurrence and prognosis. They were clinical indicators that could well reflect tumor activity and invasion ability. [31-35] Then our study further analyzed the correlation between RDW and tumor serum markers including CEA and CA19-9 and found that RDW-CV was correlated with CEA and CA19-9 in abnormal status. CEA obtained clear linear positive correlation in the analysis of continuous variables with RDW-CV particularly. When cancer cells were active in reproduction, changes in the inflammatory microenvironment could lead to abnormal status in blood. [36, 37] When tumor progression or cell proliferation was active, not only CEA and CA19-9 and other indicators were abnormal, but RDW values beyond the normal range was also reflected in the blood test results, so patients with colorectal cancer were prone to both abnormal high values of tumor markers and RDW-CV in blood. Previous reports by Wei T, et al [17] had also found that RDW was significantly correlated with the levels of CEA and CA19-9 in gastric cancer. Through our study, we found that the correlation of RDW-CV with these tumor markers could be one of the evidences to evaluate the degree of malignancy.

Studies in lung cancer have shown that RDW was associated with various indicators of inflammatory and nutritional status in patients with lung cancer and was an indicator of prognostic risk in lung cancer patients. [15, 16] According to our study's follow-up on the samples, RDW-CV was associated with DFS and OS at both three-year and 5-year follow-up points, and patients with high values were more likely to have adverse outcomes of recurrence or death. At the same time, we found that although RDW-SD was not clearly associated with DFS, but its relevance to OS had been found in our study. The above results suggest that $\mathrm{RDW}-\mathrm{CV}$ has an independent role in assessing the risk of colorectal cancer recurrence and death, and other studies have also supported this conclusion. ${ }^{[38,39]}$ Interestingly, it had been reported that the high value of RDW-CV combined with CEA can be used as an effective diagnostic indicator to screen the incidence of colorectal cancer, ${ }^{[29]}$ which suggested that we could find more accurate evaluation factors for prognosis through the combination of multiple indicators. By drawing ROC curve, we found that when RDW-CV and CEA jointly serve as evaluation indicators, their common high value indicates that AUC area with poor prognosis is larger, which meant the sensitivity and specificity were more satisfactory. Although the independent predictive ability of TNM staging was stronger in the results, it could only be obtained by experimental observation of the tumor after surgery. But blood indicators such as RDW and CEA are easier to obtain, cost less and can be monitored. Due to the above analysis results, we believe that RDW-CV combined with CEA was a more effective early warning indicator for adverse prognosis.

Blood indicators can reflect the changes of patients' whole body status, which is of great significance to diagnosis, treatment and prognosis. In addition, due to the convenience of testing, preoperative detection and low cost, more and more attention has been paid to blood markers for colorectal cancer in recent years. [13, 40-42] There have been a great number of previous studies on RDW in colorectal cancers, however, their conclusions are more inclined to the role of RDW in early cancer screening or independent effect in prognosis. In our current study, it had been found that the combined evaluation of RDW and tumor marker CEA was a more significant warning factor of prognosis. [29, 38, 39, 43-46] Although our study had reached certain conclusions, there were still some defects, such as small sample size, no survival time curve, and the detection indicators were limited to the tumor markers that have been applied in clinical practice. In another later study, we hope to conduct a more detailed analysis and proof by combining the data of molecular markers in tumor tissues by immunohistochemistry and more indicators in serum.

In conclusion, according to the findings of our report, we believe that $\mathrm{RDW}-\mathrm{CV}$ has a suggestive effect on the malignant degree of colorectal cancer and the potential function to effectively predict the prognostic risk is worthy of attention combined with CEA. The changes in RDW-CV need to be noted in clinical work, because they can be helpful for early warning and follow-up monitoring of colorectal cancers. 


\section{Conclusion}

Abnormal increase of RDW-CV in patients with colorectal cancer is not only associated with advanced age (>62 years old), infiltrated serosa, lymph node metastasis, stage III and IV, and adhesion of surrounding tissues, but also positively correlated with CEA and CA19-9.More importantly, the abnormal increase of RDW-CV was associated with poor prognosis and survival of patients and significantly decreased OS and DFS of patients with increased CEA. It is suggested that RDW can not only be used as a molecular marker for early warning of colorectal cancer patients, but also could be combined with CEA for evaluation indicators of patients' DFS and OS, and for individual prognosis evaluation of patients.

\section{Supplementary Material}

Supplementary tables.

http://www.jcancer.org/v10p1162s1.pdf

\section{Acknowledgements}

We gratefully appreciate the efforts and contributions of doctors, nurses and technical staff at the First Hospital of China Medical University.

\section{Funding}

The present study was supported in part by a grant from the Liaoning Province Education Department Foundation (No. LS201617, LK201646).

\section{Availability of data and materials}

The datasets used during the present study are available from the corresponding author upon reasonable request.

\section{Authors' contributions}

Y.L participated in literature search, study design, data collection, data analysis, data interpretation, and wrote the manuscript. Z.L, C.X, M.W, H.W, participated in study design and provided the critical revision. X.H, S.L, G.S, G.Z, B.W and F.Z carried out the data collection and data analysis, and provided the critical revision. All authors read and approved the final manuscript and agree to be accountable for all aspects of the research in ensuring that the accuracy or integrity of any part of the work are appropriately investigated and resolved.

\section{Competing Interests}

The authors have declared that no competing interest exists.

\section{References}

1. Jandial A, Kumar S, Bhalla A, et al. Elevated Red Cell Distribution Width as a Prognostic Marker in Severe Sepsis: A Prospective Observational Study. Indian J Crit Care Med. 2017; 21(9): 552-62.

2. Usman R, Jamil M, Naveed M. High Preoperative Neutrophil-Lymphocyte Ratio (NLR) and Red Blood Cell Distribution Width (RDW) as Independent Predictors of Native Arteriovenous Fistula Failure. Ann Vasc Dis. 2017; 10(3): 205-10.

3. Sarlak H, Arslan E, Cakar M, et al. Relation between unconjugated bilirubin and RDW, neutrophil to lymphocyte ratio, platelet to lymphocyte ratio in Gilbert's syndrome. Springerplus. 2016; 5(1): 1392.

4. Williams AD, Jaroudi S, Peiris AN. Red blood cell distribution width (RDW) and its potential significance to orthopedic surgeons. J Orthop. 2018; 15(1): 52.

5. Nagajothi N, Braverman A. Elevated red cell distribution width in the diagnosis of thrombotic thrombocytopenic purpura in patients presenting with anemia and thrombocytopenia. South Med J. 2007; 100(3): 257-9.

6. Siegel RL, Miller KD, Fedewa SA, et al. Colorectal cancer statistics, 2017. CA: a cancer journal for clinicians. 2017; 67(3): 177-93.

7. Colussi D, Fabbri M, Zagari RM, et al. Lifestyle factors and risk for colorectal polyps and cancer at index colonoscopy in a FIT-positive screening population. United European Gastroenterol J. 2018; 6(6): 935-42.

8. Ahmed Farag AF, Elbarmelgi MY, Azim HA, et al. TNMF versus TNM in staging of colorectal cancer. Int J Surg. 2016; 27: 147-50.

9. Topdagi O, Timuroglu A. Evaluation of the Relationship between Carcinoembryonic Antigen and TNM Stage in Colorectal Cancer. Eurasian J Med. 2018; 50(2): 96-8.

10. Browell DA, Lennard TW. Immunologic status of the cancer patient and the effects of blood transfusion on antitumor responses. Curr Opin Gen Surg. 1993: 325-33.

11. Nakano K, Sugiyama K, Satoh H, et al. Risk factors for disseminated intravascular coagulation in patients with lung cancer. Thorac Cancer. 2018; 9(8): 931-38.

12. Panova-Noeva M, Schulz A, Arnold N, et al. Coagulation and inflammation in long-term cancer survivors: results from the adult population. J Thromb Haemost. 2018; 16(4): 699-708.

13. Gao $Y$, Wang J, Zhou $Y$, et al. Evaluation of Serum CEA, CA19-9, CA72-4, CA125 and Ferritin as Diagnostic Markers and Factors of Clinical Parameters for Colorectal Cancer. Sci Rep. 2018; 8(1): 2732.

14. Li B, You Z, Xiong XZ, et al. Elevated red blood cell distribution width predicts poor prognosis in hilar cholangiocarcinoma. Oncotarget. 2017; 8(65): 109468-77.

15. Kos M, Hocazade C, Kos FT, et al. Evaluation of the effects of red blood cell distribution width on survival in lung cancer patients. Contemp Oncol (Pozn). 2016; 20(2): 153-7.

16. Koma Y, Onishi A, Matsuoka H, et al. Increased red blood cell distribution width associates with cancer stage and prognosis in patients with lung cancer. PloS one. 2013; 8(11): e80240.

17. Wei TT, Wang LL, Yin JR, et al. Relationship between red blood cell distribution width, bilirubin, and clinical characteristics of patients with gastric cancer. Int J Lab Hematol. 2017; 39(5): 497-501.

18. Benson $\mathrm{AB}, 3 \mathrm{rd}$, Venook $\mathrm{AP}, \mathrm{Al}$-Hawary $\mathrm{MM}$, et al. NCCN Guidelines Insights: Colon Cancer, Version 2.2018. J Natl Compr Canc Netw. 2018; 16(4): 359-69.

19. Benson $\mathrm{AB}, 3$ rd, Venook AP, Al-Hawary MM, et al. Rectal Cancer, Version 2.2018, NCCN Clinical Practice Guidelines in Oncology. J Natl Compr Canc Netw. 2018; 16(7): 874-901.

20. Weiser MR. AJCC 8th Edition: Colorectal Cancer. Ann Surg Oncol. 2018; 25(6): 1454-5.

21. Di Somma S, Magrini L, Travaglino F, et al. Opinion paper on innovative approach of biomarkers for infectious diseases and sepsis management in the emergency department. Clin Chem Lab Med. 2013; 51(6): 1167-75.

22. Mahmood NA, Mathew J, Kang B, et al. Broadening of the red blood cell distribution width is associated with increased severity of illness in patients with sepsis. Int J Crit Illn Inj Sci. 2014; 4(4): 278-82.

23. Sargento L, Simoes AV, Longo S, et al. Red blood cell distribution width is a survival predictor beyond anemia and Nt-ProBNP in stable optimally medicated heart failure with reduced ejection fraction outpatients. Clin Hemorheol Microcirc. 2017; 65(2): 185-94.

24. Zhao L, Mao ZG, Jiang H, et al. [Value of MCV/RDW Combined with Reticulocyte Parameters in Differential Diagnosis of Anemia Diseases]. Zhongguo Shi Yan Xue Ye Xue Za Zhi. 2015; 23(6): 1662-6.

25. Hyngstrom JR, Hu CY, Xing Y, et al. Clinicopathology and outcomes for mucinous and signet ring colorectal adenocarcinoma: analysis from the National Cancer Data Base. Ann Surg Oncol. 2012; 19(9): 2814-21.

26. Kong $X$, Zhang $X$, Huang $Y$, et al. Characteristics and prognostic factors of colorectal mucinous adenocarcinoma with signet ring cells. Cancer Manag Res. 2017; 9: 573-80.

27. Numata M, Shiozawa M, Watanabe T, et al. The clinicopathological features of colorectal mucinous adenocarcinoma and a therapeutic strategy for the disease. World J Surg Oncol. 2012; 10: 109.

28. Song $\mathrm{W}, \mathrm{Wu} \mathrm{SJ}, \mathrm{He} \mathrm{YL}$, et al. Clinicopathologic features and survival of patients with colorectal mucinous, signet-ring cell or non-mucinous adenocarcinoma: experience at an institution in southern China. Chin Med J (Engl). 2009; 122(13): 1486-91. 
29. Yang D, Quan $\mathrm{W}, \mathrm{Wu}$ J, et al. The value of red blood cell distribution width in diagnosis of patients with colorectal cancer. Clin Chim Acta. 2018; 479: 98-102.

30. Kemal Y, Demirag G, Bas B, et al. The value of red blood cell distribution width in endometrial cancer. Clin Chem Lab Med. 2015; 53(5): 823-7.

31. Nicholson BD, Shinkins B, Pathiraja I, et al. Blood CEA levels for detecting recurrent colorectal cancer. Cochrane Database Syst Rev. 2015; (12):CD011134.

32. Nicholson BD, Shinkins B, Mant D. Blood Measurement of Carcinoembryonic Antigen Level for Detecting Recurrence of Colorectal Cancer. JAMA. 2016; 316(12): 1310-1.

33. Zhai H, Huang J, Yang C, et al. Serum CEA and CA19-9 Levels are Associated with the Presence and Severity of Colorectal Neoplasia. Clin Lab. 2018; 64(3): 351-6.

34. Stojkovic Lalosevic M, Stankovic S, Stojkovic M, et al. Can preoperative CEA and CA19-9 serum concentrations suggest metastatic disease in colorectal cancer patients?. Hell J Nucl Med. 2017; 20(1): 41-5.

35. Yang SH, Jiang JK, Chang SC, et al. Clinical significance of CA19-9 in the follow-up of colorectal cancer patients with elevated preoperative serum CA19-9. Hepatogastroenterology. 2013; 60(125): 1021-7.

36. Sui $\mathrm{X}$, Lei $\mathrm{L}, \mathrm{Chen} \mathrm{L}$, et al. Inflammatory microenvironment in the initiation and progression of bladder cancer. Oncotarget. 2017; 8(54): 93279-94.

37. Kayipmaz AE, Findik M, Kavalci C, et al. Red Blood Cell Distribution Width Increases During Infection in Renal and Liver Graft Recipients. Exp Clin Transplant. 2017; 15(1): 61-4.

38. Zhang $\mathrm{X}, \mathrm{Wu} \mathrm{Q}, \mathrm{Hu} \mathrm{T}$, et al. Elevated red blood cell distribution width contributes to poor prognosis in patients undergoing resection for nonmetastatic rectal cancer. Medicine (Baltimore). 2018; 97(3): e9641.

39. Han F, Shang X, Wan F, et al. Clinical value of the preoperative neutrophil-to-lymphocyte ratio and red blood cell distribution width in patients with colorectal carcinoma. Oncol Lett. 2018; 15(3): 3339-49.

40. Nikolaou S, Qiu S, Fiorentino F, et al. Systematic review of blood diagnostic markers in colorectal cancer. Tech Coloproctol. 2018; 22(7): 481-98.

41. Fung KY, Tabor B, Buckley MJ, et al. Blood-based protein biomarker panel for the detection of colorectal cancer. PLoS One. 2015; 10(3): e0120425.

42. Zhao JX, Liu LR, Yang XY, et al. Serum CA19-9 as a marker of circulating tumor cells in first reflux blood of colorectal cancer patients. Oncotarget. 2017; 8(40): 67918-32.

43. Pilling LC, Atkins JL, Kuchel GA, et al. Red cell distribution width and common disease onsets in 240,477 healthy volunteers followed for up to 9 years. PLoS One. 2018; 13(9): e0203504.

44. Kust D, Lucijanic M, Urch K, et al. Clinical and prognostic significance of anisocytosis measured as a red cell distribution width in patients with colorectal cancer. QJM. 2017; 110(6): 361-7.

45. Ay S, Eryilmaz MA, Aksoy N, et al. Is early detection of colon cancer possible with red blood cell distribution width?. Asian Pacific journal of cancer prevention : APJCP. 2015; 16(2): 753-6.

46. Montagnana M, Danese E. Red cell distribution width and cancer. Ann Transl Med. 2016; 4(20): 399. 